\title{
COMPLICAÇÕES DO DIABETES MELLITUS
}

\author{
COMPLICATIONS OF DIABETES MELLITUS
}

\author{
Fonseca, Kathlem Pereira ${ }^{1}$, Rached, Chennyfer Dobbins $\mathrm{Abi}^{2}$
}

1 Enfermeira. Especialista em Estomaterapia. Faculdade Unyleya - DF - Brasil

\begin{abstract}
2 Doutora em Saúde Coletiva; Mestre em Economia da Saúde pela Universidade Federal de São Paulo (UNIFESP); Docente do Programa de Mestrado Profissional em Gestão em Sistemas de Saúde - Universidade Nove de Julho - UNINOVE

Orcid: https://orcid.org/0000-0002-4499-3716 email: chennyferr@yahoo.com.br
\end{abstract}

\begin{abstract}
Resumo
Entre as complicações da DM a neuropatia diabética é a de maior incidência a longo prazo, sendo as alterações sensoriais e motoras as de maior impacto, pelo fato de levar a manifestação do conhecido Pé Diabético. Trata-se de uma das mais graves e onerosas complicações do DM, e uma amputação de extremidades inferior ou parte dela e geralmente consequência de uma ulcera no pé. É um trabalho com base em uma revisão bibliográfica de literatura em narrativa, baseado em evidências cientificas com a coletas de dados de artigos, revistas nacionais. Com o objetivo de compreender a importância da orientação da enfermagem diante das complicações do Diabetes Mellitus e conceituar o tema pé diabético É de grande necessidade que os profissionais de saúde avaliem os pés das pessoas com diabetes de forma minuciosa e com frequência regular, desenvolvendo atividades educativas, para melhorar o autocuidado, principalmente a manutenção de um bom controle glicêmico. O presente estudo se dá devido aos altos números de pacientes portadores de diabetes e que por falta de cuidados dos mesmos estão levando ao pé diabético e outras complicações. Discorre sobre a importância da assistência de enfermagem diante das complicações do Diabetes Mellitus.
\end{abstract}

Palavras-Chave: Diabetes Mellitus; Infecção Hospitalar; Saúde Coletiva.

\begin{abstract}
Among DM complications, a diabetic neuropathy is the highest incidence in the short term, with sensory and motor associations as the greatest impact, as it leads to the manifestation of the known Diabetic Foot. It is one of the most serious and costly complications of DM, and an amputation of lower extremities or part of it and consequence of an ulcer in the foot. It is a work based on a bibliographical review of literature in narrative, based on scientific evidences with the data collection of articles, national journals. With the objective of evaluating nursing accounting in the face of complications of Diabetes Mellitus and conceptualizing the topic of diabetic foot It is of great necessity for health professionals to evaluate the feet of people with diabetes in a thorough and regular way, developing educational activities, to improve self-care, especially maintaining good glycemic control. Notices are easy to evaluate and can help. Discusses the importance of nursing care in the face of complications of Diabetes Mellitus: diabetic foot
\end{abstract}

Keywords: Diabetes Mellitus; Infection; Public Health. 


\section{Introdução}

O diabetes é um problema de saúde altamente prevalente em nosso meio e considerado como uma das doenças de mais difícil controle, por ser crônica, sistêmica e multifatorial. Trata se de um distúrbio metabólico causado pela completa ou parcial deficiência de insulina pelo pâncreas e ou diminuição de sua ação nos tecidos prejudicando o metabolismo dos lipídeos, glicídios, proteínas, água, vitaminas e minerais (Torres, Fernandes e Cruz,2007 e Hashimoto e HADDAD,2009).

E de grande necessidade que os profissionais de saúde avaliem os pés das pessoas com diabetes de forma minuciosa e com frequência regular, desenvolvendo atividades educativas, para melhorar o autocuidado, principalmente a manutenção de um bom controle glicêmico, presente estudo justifica se por sua relevância acadêmica e chama atenção por altos números de pacientes portadores de diabetes e que por falta de cuidados dos mesmos estão levando a futuras complicações da doença.

E responder a seguinte pergunta qual a importância da orientação da enfermagem diante das complicações do Diabetes Mellitus: Pé Diabético? Então consulta de enfermagem se torna de suma importância no procedimento de trabalho para o acolhimento destes pacientes, o profissional de enfermagem e sua equipe devem desenvolver suas atividades e ações visando a prevenção. E deve ser efetivado dentro de um sistema hierarquizado de assistência com base na atenção primária.

$\mathrm{Na}$ consulta de enfermagem, o enfermeiro deve realizar a anamnese e o exame físico de forma frequente e minuciosa para detectar possíveis agravos, mas por muitas vezes e negligenciado gerando mais gastos para a rede de saúde e sequelas a esse paciente, por isso e pertinente o estudo sobre o tema.

O presente estudo tem como objetivo compreender a importância da orientação da enfermagem diante das complicações do Diabetes Mellitus: conceituar o tema pé diabético, a avalição dos pés destes pacientes visando evidenciar as complicações causadas pelo Diabetes Mellitus; Apresentando a falta de conhecimento a falta de conhecimento dos pacientes portadores de Diabetes Mellitus e em relação aos cuidados com os pés.

A escolha deste assunto se deu pelo fato do pouco conhecimento e adesão ao tratamento correto da patologia, por falta de conhecimento, orientação e conscientização. Diminuindo assim a qualidade de vida ou até mesmo o tempo de vida do paciente.

O cliente bem informado tem condições de seguir de forma correta um tratamento, pois já está conscientizado que tem a doença.

Trata-se de uma revisão bibliográfica de literatura em narrativa, baseado em evidências cientificas com a coletas de dados de artigos, revistas nacionais disponíveis sendo os principais LILACS e SCIELO. E livros e manuais de acordo com as referências citadas. Tendo como principais características a análise de informações e estudos para a revisão de literatura e busca, conhecimento e elaboração de uma resposta de acordo com os conhecimentos adquiridos.

O referencial teórico será embasado nas literaturas utilizadas, e artigos científicos em textos publicados nos Manuais do Ministério Público de Saúde, Leis e Diretrizes do Profissional de Enfermagem e em bibliografia pertinente onde foi feito o pré-projeto e posteriormente a construção do trabalho.

Para análise e síntese destes foi realizada uma leitura exploratória para reconhecer do que se tratava o texto, foi selecionando o material em busca de informações relacionado aos objetivos e o tema 
proposto. Desta forma, acredita-se que com isso que o trabalho seja de ampla relevância social, para a enfermagem e pacientes, já que, através da intervenção, sistematização e da educação em saúde é possível capacitar e sensibilizar os profissionais quanto aos agravos do diabético e ao atendimento deste cliente.

\section{Objetivo}

Apresentar uma revisão da literatura quanto as complicações e cuidados para o paciente com diabetes mellitus.

\section{Método}

Trata-se de uma revisão narrativa da literatura.

\section{REVISÃO DE LITERATURA}

O diabetes mellitus é um conjunto de doenças que provocam a hiperglicemia por deficiência de insulina. Essa deficiência pode ser absoluta, por baixa produção, ou relativa devido à resistência periférica a insulina.

A forma mais frequente da doença, o DM tipo 2 ou DM do adulto é uma doença crônica silenciosa no início responsável por uma série de complicações (PEDROSO; OLIVEIRA 2007). Estima -se que um nível global, a prevalência do DM, seja em torno de 120 milhões de indivíduos e que, de 4 a 10\% desenvolvam lesões nos pés.

Trata-se de uma complicação que ocorre em média, após 10 anos de evolução dessa doença, tornandose a causa mais comum de amputações não traumáticas. (HADDAD et al.;2008).

A prevalência do DM é estimada em 11\% da população (IBGE), acima de 40 anos, segundo dados de pesquisa de prevalência de 1986 e que vem sendo usada 18 como parâmetro para a política nacional de atenção integral à hipertensão arterial e a Diabetes Mellitus pelo ministério da saúde. (Prefeitura do Rio de Janeiro de 2010).

Observa -se o aumento e agravo da DM e que está representando um alto índice de problema de saúde pública. Problema esse que afeta a qualidade de vida, suas atividades diárias, psicológicas e sociais do indivíduo com essa patologia.

O DM é a principal causa de cegueira entre 20 e 74 anos de idade a principal causa de amputações e a principal causa de insuficiência renal, sendo responsável por $44 \%$ dos casos de hemodiálise (PREFEITURA DO RIO DE JANEIRO 2010).

Uma das complicações que merece destaque diz respeito ao Pé Diabético, que trata-se de uma das mais graves e numerosas complicações do DM, é uma amputação de extremidades inferior ou parte dela e geralmente consequência de uma úlcera no pé.

O Pé Diabético é o conjunto de alterações ocorrida no portador de DM, decorrente de neuropatias, micro e macrovasculopatia e aumento da susceptibilidade a infecção, devido às alterações biomecânicas que levam à deformidade (PACE; VIGO 2004). 
Estudos clínicos bem controlados demonstram que o bom controle da doença e capaz de prevenir ou retardar o aparecimento de tais complicações (Prefeitura do Rio de Janeiro,2010). O diagnóstico do DM ou acompanhamento e verificado através das próprias manifestações clínicas do paciente, eles relatam o histórico familiar, sempre os mesmos relatam obesidade, sedentarismo, tabagismo.

O diagnóstico correto é precoce do DM do tipo 2 permite que sejam adotadas medidas terapêuticas que podem evitar e ou retardar o aparecimento das complicações crônicas, principalmente as cardiovasculares nos pacientes diagnosticados com diabetes (LUCAS ET AL.,2010). Quando o diagnóstico de DM é estabelecido os 60 anos de idade, o indivíduo poderá ainda viver o bastante para que uma complicação crônica se instale, considerando que muitas vezes, não se sabe com exatidão o tempo de diagnóstico da doença. (BRASIL, 2013)

Também existe o consenso de que a maioria das complicações crônicas do DM apresenta progressão acelerada no idoso e, portanto, o bom controle glicêmico deve ser perseguido com maior tenacidade (Hashimoto e Haddad,2009).

A atuação do Enfermeiro junto a equipe de saúde é muito importante no sentido de orientar 19 pacientes diabéticos sobre os cuidados diários com os pés e a prevenção do aparecimento de úlceras, conscientizando que seus pés são sensíveis e por isso, devem evitar traumas seja mecânico, químico ou térmico. Por meio de adoção de medidas simples, como a higienização, hidratação e o uso de sapatos adequados (GIL, HADDAD E GUARIENTE, HIROTA, 2008).

\section{DIABETES MELLITUS TIPO 1}

Acomete cerca de 5 a 10\% dos portadores de DM, e geralmente tem início em indivíduos com menos de 30 anos de idade, entretanto pode acometer pessoas em qualquer faixa etária.

Ocorre devido à destruição das células beta do pâncreas, geralmente por processo autoimune, determinada forma autoimune tipo $1 \mathrm{~A}$, ou, de forma menos frequente, por causa desconhecida, determinada forma idiopática tipo 1B (GROSS, et al. 2016). Nestas formas de DM, a taxa de destruição das células beta do pâncreas ocorrem de maneira variável, podendo ser rápida em alguns indivíduos, como em crianças, por exemplo, e lenta em outros, como nos adultos, por exemplo.

Geralmente, a primeira manifestação da doença é a cetoacidose, principalmente em crianças e adolescentes, e expressa diretamente à deficiência de insulina, mas também pode estar associada à presença de estresse infeccioso ou uso inadequado de insulina (ADA, 2004).

Ainda nessa classificação temos a diabetes autoimune latente do adulto (LADA), que engloba pacientes adultos, geralmente com mais de 30 anos, que de início não requerem insulina, porém possuem auto anticorpos contra as células beta do pâncreas, e que evoluem rapidamente, cerca de seis meses após o início, levando a necessidade do uso de insulina (CALSOLARI, et al. 2008).

\section{DIABETES MELLITUS TIPO 2}


É a forma mais comum de DM, acometendo cerca de $90 \%$ dos pacientes diabéticos, e resulta da deficiência da secreção de insulina ou de sua ação, podendo culminar em um aumento da produção hepática de glicose, decorrentes dessas alterações em torno da insulina.

A predisposição para ocorrência de DM tipo 2 está interligada entre fatores genéticos e ambientais, onde o estilo de vida é um dos fatores principais para o seu desencadeamento (CHAVES; ROMALDINI, 2002).

Geralmente seu desenvolvimento é lento, principalmente nas fases iniciais da doença, o que faz com que essa forma de diabetes permaneça por muitos anos sem diagnóstico, devido ao desenvolvimento gradativo da hiperglicemia e a ausência de sintomas característicos. Isso potencializa as chances de agravamento da doença, uma vez que o diagnóstico geralmente é tardio (GUIMARÃES; TAKAYANAGUI, 2002).

A cetoacidose é uma complicação característica da DM tipo 1, mas pode acometer portadores de DM tipo 2 em situações de estresse para o organismo.

Na DM tipo 2, o aparecimento da DM pode ocorrer tardiamente e de forma mais lenta, porém, esse aparecimento tardio também pode ocorrer em pacientes com DM tipo 1.

Esses fatores dificultam definir com clareza o diagnóstico entre DM tipo 1 e DM tipo 2 (MEIRELES, et al. 2013).

\section{DIABETES GESTACIONAL}

É considerado Diabetes Gestacional (DG) o aparecimento de qualquer grau de intolerância à glicose que se manifeste durante a gravidez, ou que tenha o seu reconhecimento pela primeira vez no período de gestação (QUEIRÓS; MAGALHÃES; MEDINA, 2006).

Representa riscos tanto para a mãe quanto para o bebê, e a probabilidade de que as mulheres que desenvolveram DG se tornem diabéticas futuramente são consideráveis (WEINERT, et al. 2016).

\section{COMPLICAÇÕES ASSOCIADAS À DIABETES MELLITUS}

O estilo de vida do paciente diabético, incluindo fatores como sedentarismo, alimentação e até mesmo a maneira que ele controla os seus níveis glicêmicos através do tratamento, influenciam nas complicações advindas do DM. Visto isso, é de extrema importância o controle dos níveis glicêmicos, uma vez que a persistência dessa hiperglicemia pode culminar em complicações agudas, como, cetoacidose diabética, coma hiperosmolar não-cetótico e hipoglicemia, quanto complicações crônicas, como as microvasculares (neuropatia periférica, retinopatia e nefropatia) e macrovasculares (doença arterial coronariana, doença cerebrovascular e vascular periférica).

Ambas as complicações estão relacionadas ao tempo da doença, onde a aguda tem a manifestação de seus sintomas de forma mais imediata, e a crônica provém de uma manifestação dos seus sintomas após anos de evolução da doença, e que se relacionam diretamente a um controle glicêmico inadequado (MORAIS, et al. 2009). 


\section{NEUROPATIA AUTONÔMICA DIABÉTICA}

A neuropatia autonômica diabética (NAD) possui maior prevalência para complicação crônica, podendo atingir cerca de $40 \%$ dos pacientes com DM. Não é considerada causa de morte, porém, na fase avançada da doença, contribui muito para uma incapacitação do indivíduo.

A NAD afeta sistemas como o cardiovascular, urogenital, digestivo, glandular, e ainda compromete a motricidade pupilar. $\mathrm{Na}$ sua grande maioria, os pacientes com NAD geralmente apresentam retinopatia (FOSSFREITAS; JUNIOR; FOS, 2008).

\section{RETINOPATIA DIABÉTICA}

A retinopatia diabética é uma complicação ocular severa, que em estados avançados, se torna uma das principais causas de cegueira irreversível. Na maioria das vezes, se manifesta de forma tardia, aumentando progressivamente com o decorrer da doença, em especial em pacientes com DM tipo 1, que após cerca de 20 anos de duração da DM, apresentam um risco maior de desenvolver algum grau de retinopatia. Por ser irreversível, a retinopatia não tem cura, e os esforços terapêuticos baseiam-se na observação e prevenção dos fatores de risco para o aparecimento e agravamento da retinopatia (BOELTER, et al. 2003).

\section{NEFROPATIA DIABÉTICA}

A nefropatia diabética ocorre tanto no DM tipo 1, quanto no DM tipo 2, sendo mais prevalente em pacientes com DM tipo 2.

Nesta complicação, o processo de filtração glomerular é inadequado, levando a excreção de moléculas de proteínas com baixo peso molecular pela urina, tornando-se um marcador importante para o diagnóstico desse tipo de complicação.

A nefropatia diabética é uma das principais causas de insuficiência renal em pacientes que estão realizando diálise e é caracterizado por albuminúria, HAS e um declínio progressivo da função renal (PASQUALOTTO, et al. 2012).

\section{MIOCARDIOPATIA DIABÉTICA}

A miocardiopatia diabética é desencadeada pela diabetes, sendo uma doença que provoca necrose, apoptose e hipertrofia do músculo cardíaco, devido às anormalidades metabólicas causadas pela diabetes, como hiperinsulinemia nas fases mais precoces e hiperglicemia após a falência das células beta pancreáticas.

Essas alterações podem aumentar o estresse oxidativo e levar a perda celular. O diagnóstico se torna difícil de ser estabelecido, uma vez que os sintomas e achados de exames são inespecíficos (OKOSHI, et al. 2006).

\section{DISLIPIDEMIA}


A dislipidemia é caracterizada pela Hiperglicemia e resistência à insulina, a qual favorece o desenvolvimento de aterosclerose por uma disfunção no endotélio devido à diminuição do coesterol HDL e aumento do LDL e triglicerídeos. Esse fator leva o indivíduo ao risco doença cardiovascular (ALMEIDA et al., 2007).

Para Rodrigues e Motta (2012), o Diabetes Mellitus ocorre pelo aumento da glicemia com alterações na metabolização da glicose. Na presença de complicações, ocorre a inversão metabólica, com a degradação da proteína e dos lipídeos, decorrente da falta da insulina, podendo acometer órgãos importantes reduzindo a qualidade de vida do indivíduo

\section{PÉ DIABÉTICO}

O pé diabético é uma das complicações crônicas causada pelo mau controle da diabetes que mais causa internações, caracterizada por lesões nos pés desencadeadas devido a alterações vasculares periféricas e/ou neurológicas, em decorrência da diabetes mellitus, baseada na tríade: neuropatia, doença vascular periférica e infecção.

O fator mais importante que leva a formação de úlceras nos membros inferiores é a neuropatia diabética (SANTOS, et al. 2011).

Alguns fatores contribuem para a ocorrência do pé diabético, como idade, controle inadequado de glicemia, tipo e tempo de diagnóstico, obesidade, HAS e falta de hábitos de higiene e cuidados com os pés. É importante ressaltar que a neuropatia diabética leva a uma perda da sensibilidade dos membros inferiores, o que torna susceptível o acontecimento de lesões, sendo uma porta de entrada para infecções, que caso não tratadas precocemente, podem levar a amputação do membro (BRASILEIRO, et al. 2004).

Dentre as complicações incapacitantes do diabetes mellitus, destaca-se a neuropatia diabética periférica que é a complicação mais frequente e também chamada de pé diabético, quando associada com vasculopatia nos membros inferiores. (MANTOVANI ET. AL. 2013).

A função da enfermagem resume-se em ao cuidado, que tem como objetivo a integralidade daquele paciente/cliente que naquele momento está debilitado e fragilizado.

O Enfermeiro deverá oferecer uma assistência de qualidade e observar agravantes a saúde de seus pacientes e também estar acompanhando o controle da sua patologia. (BRASIL, 2016) Fatores estes que irão contribuir como importantíssimas ações de prevenção para o não surgimento do pé diabético e outros agravos da doença.

A atuação do Enfermeiro junto a equipe de saúde é importante no intuito de orientar os pacientes diabéticos com cuidados diários com a alimentação e também com lesões já bem avançadas. Em todos os tipos de tratamento a atuação do Enfermeiro é importante, porque são eles que estão em constante contato com os pacientes realizando curativos, assistindo a evolução clinica das feridas e também os apoiando psicologicamente. (BRASIL, 2013)

O termo Diabetes Mellitus aplica -se a um grupo de distúrbios metabólicos, de etiologia múltipla, que se caracterizam bioquimicamente, por hiperglicemia crônica e clinicamente, pelo 
desenvolvimento a longo prazo de complicações microvasculares (DIAS, SOARES; RESENDE, 2002.p.341).

A Diabete Mellitus é um dos problemas mais importantes de saúde, tanto em número de pessoas afetadas, incapacitadas, mortalidade, custos envolvidos no controle e também no tratamento de suas complicações.

Termos de números da doença há cerca de 246 milhões de pessoas no mundo inteiro. Estima que até 2025 a previsão que esse número chegue a 380 milhões e que uma parte de pessoas que tem diabetes desconhece a sua própria condição devido à falta de interesse.

E a grande maioria só irá descobrir a doença na hora de haver uma complicação e muitas vezes os danos já são irreparáveis ao organismo. No Brasil a diabetes mellitus há altos índices de mortalidade sendo uma das cinco causas principais. Elas acometem principalmente o sistema cardiovascular e também o sistema nervoso. Na alteração presente existem três tipos de pé diabético: Neuropaticoalteraçoes dos nervos sensitivos, motores e simpáticos. (BRASIL, 2013) Microangiopatico que são alterações da microcirculação. Isquêmico que são alterações da microcirculação. As pesquisas clinicas no pé diabético infeccioso são: infecção aos tecidos moles, associada ou não a osteomielite (inflamação nos ossos), gangrena úmida, celulite necromante e abcesso. As bactérias mais encontradas são as sthaphilococus aureos, streptococcus aureos, streptococus sp e o gram negativo. Segundo Dias, Soares e Resende (2002):

As pessoas com diabetes têm maior dificuldade de cicatrização sim, por serem pessoas com microangiopatias, isto é, que já tem lesão de pequenos vasos e problemas de irrigação. Além disso, mais propensas a contrair infecções. Entretanto, a dificuldade de cicatrização não é um problema que contra indique qualquer procedimento no diabético. (DIAS, SOARES; RESENDE, 2002.p.341).

\section{OUTRAS COMPLICAÇÕES}

\section{HIPERTENÇÃO ARTERIAL}

A Hipertensão Arterial pode ser detectada no paciente portador de Diabetes Mellitus, sendo uma condição clínica, que em muitos casos, o paciente, quando 60 diagnosticado com diabetes, já se encontra hipertenso. Esta associação aumenta o risco de complicações no indivíduo. (SOCIEDADE BRASILEIRA DEDIABETES, 2009).

Segundo Carvalho,A. e outros (2012) aumentou o número de doenças não transmissíveis em pessoas com Hipertensão Arterial e Diabetes Mellitus e as duas juntas acometem ao mesmo tempo a pessoa. Essas doenças são causadas pelos maus hábitos de vida, através dos quais o indivíduo fica sedentário e, com isso, chega à obesidade.

Para os autores há uma tendência de que a hipertensão e o diabetes se tornem crônicas por, às vezes, serem assintomáticas, com perigo para problemas cardiovasculares que podem levar ao risco de morte, gerando um grande impacto na saúde e na sociedade.

Com os dados obtidos, o Sistema de Saúde e os órgãos responsáveis devem adotar medidas para prevenção destas doenças na atenção primária ao paciente. O controle da pressão arterial é importante 
para que o indivíduo com diabetes não acelere o desenvolvimento das complicações, uma vez que está diretamente relacionada a este evento.

O aumento da pressão arterial é preocupante por elevar o risco da mortalidade por problemas cardiovasculares e renais. $\mathrm{O}$ controle com anti hipertensivos deve ser monitorado devido à disfunção renal (GROSS et al., 2007).

\section{DEPRESSÃO}

Segundo o estudo de Nascimento e outros (2010), o Diabetes de Mellitus é uma doença grave e crônica que traz complicações em longo prazo. Uma das complicações é a depressão que acomete o paciente com Diabetes de Mellitus tipo 2 e pode ser desencadeada pelo uso excessivo de medicamentos que são necessários para o controle da doença.

Conforme os autores, através de estudo com paciente portadores do Diabetes Mellitus tipo 2, foi identificado que o indivíduo está propenso a desenvolver depressão tanto no impacto que o Diabetes de Mellitus traz para a vida, bem como no uso de vários medicamentos que ativam agentes estressores, os quais aumentam a liberação de substâncias responsáveis por ativar a depressão.

A depressão é um transtorno que pode estar relacionados com as complicações do Diabetes Mellitus ou com as limitações que atingem os pacientes e grande parte de diabéticos com depressão não recebem o diagnóstico e tratamento adequado (SOCIEDADE BRASILEIRA DE DIABETES, 2009).

\section{DOENÇA PERIODENTAL}

A doença periodental é uma complicação que pode iniciar mesmo quando o Diabetes Mellitus não foi diagnosticado. Ocasiona alterações na boca como queilose, fissura ressecamentos de mucosas, diminuição do fluxo salivar, dificuldade de cicatrização e alteração da microbiota. Os medicamentos também podem provocar alterações na saliva (SOCIEDADE BRASILEIRA DE DIABETES, 2009).

\section{ESCLEREDEMA DE BUSCHKE}

Segundo o estudo de Dinato e outros (2010), o escleredema é uma das complicações da pele que pode acometer o indivíduo portador do Diabete Mellitus.

Essa doença acomete a pele dando a ela a característica enrijecida por ser uma alteração do tecido conjuntivo que pode se agravar e reduzir a qualidade de vida.

Apesar de ser uma doença pouco conhecida, se for diagnosticada precocemente há a possibilidade de controlar o avanço das lesões que podem se tornar graves e levar a morte. O Escleredema de Buschke foi descrito em 1902 e se apresenta em forma de lesões endurecidas na pele que causam desconforto para o paciente.

\section{GASTROENTEROPATIA DIABÉTICA}

A Gastroenteropatia Diabética é uma complicação caracterizada por alterações gastrointestinais que acometem o paciente com Diabetes Mellitus. Os sintomas são náuseas, vômitos, dor abdominal, pirose, disfagia, constipação, diarreia, sensibilidades viscerais e inflação de mucosas. Estas alterações 
gastrointestinais ocorrem no sistema nervoso entérico, com o comprometimento de todo o sistema. (RODRIGUES; MOTTA, 2012).

\section{ORIENTAÇÕES PARA A PROMOÇÃO DO AUTOCUIDADO DE PESSOAS COM DM EM RELAÇÃO AO CUIDADOS COM OS PÉS.}

Avaliar o conhecimento do paciente sobre o diabetes;

Avaliar o conhecimento sobre os cuidados com os pés e as unhas (complicações agudas e crônicas de fácil identificação);

Avaliar o comportamento do paciente com relação aos seus pés;

Avaliar o cuidado executado pela pessoa;

Avaliar o apoio familiar no cuidado com os pés;

Avaliar as condições dos calçados e das palmilhas.

\section{A IMPORTÂNCIA DA AVALIAÇÃO DOS PÉS DO DIABÉTICO}

Entre as complicações da DM a neuropatia diabética é a de maior incidência a longo prazo, sendo as alterações sensoriais e motoras as de maior impacto, pelo fato de levar a manifestação do conhecido Pé Diabético.

Segundo o $22^{\circ}$ Consenso Internacional sobre o Pé Diabético realizado em 1999, o "Pé Diabético" é a infecção, ulceração e/ou destruição de tecidos profundos associados com anormalidades neurológicas e vários graus de doença vascular periférica no membro inferior.

Existem três processos patológicos básicos que constituem o tripé que justifica a ocorrência do PD: isquemia, neuropatia e infecção.A combinação dos efeitos destes processos, em graus variáveis de paciente para paciente, causam as deformidades, úlceras, infecções e gangrena, clinicamente reunidas sob o termo "Pé Diabético" (KOZAK et al, 1996 e BRASIL, 2016).

A literatura relata outros elementos de avaliação dentro do exame físico, tais como: sinais e sintomas do paciente com pé diabético, dor em repouso, claudicação e presença de úlcera, devem ser julgados e averiguados para uma futura intervenção.

A neuropatia diabética leva à insensibilidade do membro e, e assim tem como consequência, situações que levam a amputação do pé (DONOSO; ROSA; BORGES, 2013).

Para se evitar a amputação, a maneira mais eficaz ainda é a prevenção. O diabético e seus familiares necessitam conhecer o que é o pé e saber o risco para o desenvolvimento de úlceras e ser orientado sobre os cuidados de rotina que deve ser adotado.

Alguns estudos relatam uma redução entre 44\% a 85\%, apenas com cuidados preventivos, efetivos e apropriados com os pés (HIROTA et al., 2008; AUDI et al., 2011). Por isso a importância do enfermeiro, este se encontra próximo da realidade comunitária, deve usar a avaliação clínica para descobrir achados clínicos e assim evitar sequelas graves.

São vários os testes utilizados no diagnóstico da polineuropatia: teste da sensação vibratória com diapasão de $128 \mathrm{~Hz}$, teste da sensação dolorosa com estilete, teste da sensibilidade térmica, teste da 
sensação profunda com martelo (reflexo do tendão de Aquiles), teste do monofilamento, entre outros (REIBER, 2002).

As lesões sensitivas são as mais importantes, pois a partir da diminuição da sensibilidade dolorosa o paciente passa a não mais sentir o incômodo da pressão repetitiva do calçado desconfortável, de objetos pontiagudos no chão, tesoura. E, a partir daí há maior suscetibilidade a formação de calosidades na planta dos pés e úlceras atróficas, que são responsáveis pelo início dos processos infecciosos e das gangrenas (BRASIL, 2016). Está relacionado ao desenvolvimento das alterações que caracterizam o PD está relacionado ao comprometimento das vias nervosas periféricas de controle autônomo, já relatado anteriormente, que provoca distúrbios cutâneos que fragilizam a pele e a predispõe às lesões da superfície plantar. Esta fragilidade da pele decorre de uma inadequada nutrição da pele plantar tornando a camada mais basal do tecido subcutâneo mais tênue e frágil, predispondo a pele plantar às lesões decorrentes das forças de compressão e cisalhamento exercidas pela transferência do peso corporal.

O PD é a principal complicação incapacitante consequente da DM. Atribui-se ao diabetes $50 \%$ das amputações não-traumáticas de membros inferiores, sendo o risco para amputação cerca de quinze vezes maior do que na população em geral (REIBER, 2002).

As amputações entre pessoas diabéticas são precedidas de úlceras, 22 caracterizadas por erosões cutâneas com perda do epitélio, as quais se estendem até a derme ou a atravessa chegando aos tecidos mais profundos e, algumas vezes, atingem os ossos e os músculos. Além disso, três anos após a amputação de um membro inferior, a porcentagem de sobrevida do indivíduo é de cerca de $50 \%$ (REIBER, 1996).

As lesões do PD são precipitadas por trauma e frequentemente complicam com infecção, podendo terminar em amputações quando não for instituído um tratamento precoce e adequado. As úlceras do PD são responsáveis por grande percentual de morbimortalidade e hospitalizações, ocasionando um período de internação 59\% mais prolongado que pessoas sem NDP. Estes tipos de internamentos geralmente são recorrentes, exigem grande número de consultas ambulatoriais, além da necessidade constante de cuidados domiciliares.

Estima-se que as taxas de recorrência das úlceras, em longo prazo, sejam de 44\% no primeiro, $61 \%$ no terceiro e $70 \%$ no ano (PEDROSA et al., 1998).

Segundo Andreoli (1994), as úlceras dos pés podem ocorrer em pacientes diabéticos secundariamente a aterosclerose de grandes vasos, micro angiopatia, neuropatia ou uma combinação desses fatores.

As úlceras secundárias as doenças dos grandes vasos ocorrem tipicamente nas pontas dos artelhos, enquanto as secundárias, neuropatia ocorrem em áreas de sustentação de peso e com pressão (superfície plantar).

A terapia para as úlceras dos pés de diabéticos é a prevenção, os clientes precisam ser orientados a observar regularmente os seus pés a procura de calosidade, feridas ou inflamação, e seus pés devem ser mantidos limpos e secos, além disso, os sapatos de um diabético devem ser apropriadamente ajustados. Andar descalço é perigoso e devem ser desencorajados pelo fato de causar um ferimento, 
já que possuem uma difícil cicatrização o mesmo pode aumentar e infeccionar causando uma possível úlcera.

Cautelosos cuidados com os pés podem atenuar os casos de úlceras e gangrena e evitar amputações. $\mathrm{O}$ tratamento inclui repouso na cama, elevação dos pés e desbridamento. Se houver evidências de infecção, deve ser colhido material para cultura, inclusive para anaeróbios. A terapia inicial com antibióticos deve ser efetiva contra bactérias Gram-positivas, Gram-negativas e 23 anaeróbios, com uma posterior terapia orientada pelos resultados da cultura. (BRASIL,2016)

\section{Conclusão}

Achados literários apontam a importância da prevenção que é a maior ferramenta do cuidado ao paciente diabético sendo fundamental as atividades educativas em saúde.

Deve-se sempre avaliar os pés bem como o grau da lesão e evitar que a mesma se agrave através do cuidado e orientação destes pacientes, o enfermeiro deve, assim, agir de forma holística e projetar as suas ações gerando atividades educacionais nas reuniões e consultas com diabéticos, em específico no Programa de Hipertensos e Diabéticos (HIPERDIA) da atenção básica, por meio de apresentações faladas, rodas de conversas, preparação de panfletos educativos, folders com as orientações, como métodos de precaução e de autocuidado, impedindo assim novas complicações.

Se faz necessário novas pesquisas para obtenção de meios e dados científicos que evidencie novas técnicas e cuidados com esses pacientes a respeito dos medicamentos, alimentação e cuidados que demonstre melhoras significativas para esses agravos e lesões afim de evitar amputação do membro e até morte destes pacientes.

\section{Referências}

ANDRADE, N. H. S.; MENDES, K. D. S.; FARIA, H. T. G. et al. Pacientes com diabetes mellitus: cuidados e prevenção do pé diabético em atenção primaria á saúde. Revista de Enfermagem da UERJ, Rio de Janeiro, v. 18 n. 4, 2010, p. 616-621.

AUDI, E. G.; MOREIRA, R. C.; MOREIRA, A. C. M. G. et al. Avaliação dos pés e classificação do risco para pé diabético: contribuições da enfermagem. Revista Cogitare Enfermagem, v. 16, n. 2, 2011, p. 240-246.

Brasil. Ministério da Saúde. Secretaria de Atenção à Saúde. Departamento de Atenção Básica. Manual do pé diabético : estratégias para o cuidado da pessoa com doença crônica / Ministério da Saúde, Secretaria de Atenção à Saúde, Departamento de Atenção Básica. - Brasília : Ministério da Saúde, 2016. p 62

Brasil. Ministério da Saúde. Secretaria de Atenção à Saúde. Departamento de Atenção Básica. Estratégias para o cuidado da pessoa com doença crônica : diabetes mellitus / Ministério da Saúde, Secretaria de Atenção à Saúde, Departamento de Atenção Básica. - Brasília : Ministério da Saúde, 2013. 160 p. : il. (Cadernos de Atenção Básica, n. 36)

BRANGANÇA, C. M.; GOMES I. C.; FONSECA, M. R. C. C. et al Avaliação das práticas preventivas do pé diabético. J Health Sci Inst, v. 28, n. 2, 2010, p.159-163.

BATISTA, L. L.; LOPES, C. H. A. F. Enfermeiro no cuidado do paciente com ulcera de pé diabético. Revista Baiana de Enfermagem, Salvador. v. 20, n. 1/2/3, 2006, p. 47- 55. 
DIAS, E. P; SOARES, M. M. S; RESENDE, L. M. H. Diabetes mellitus: diagnóstico e classificação. In. BRAGA, W.R.C. Enciclopédia da saúde: diabetes mellitus. Rio de Janeiro: Medsi, 2002. p. 341.

CAIFA, J. S.; CASTRO, A. A.; FIDELES, C. et al. Atenção integral ao portador de pé diabético. Jornal Vascular Brasileiro. Porto Alegre, v. 10, n. 4, 2011, p.1-32.

CARVALHO, R. D. P.; CARVALHO, C. D. P.; MARTINS, D. A. Aplicação dos cuidados com os pés entre portadores de diabetes mellitus. Revista Cogitare Enfermagem, v. 15, n. 1, 2010, p. 106109.

COELHO, M. S.; SILVA, D. M. G. V. Grupo educação-apoio: visualizando o auto cuidado com os pés de pessoas com diabetes mellitus. Revista Ciência, Cuidado e Saúde, Paraná. v. 5 n. 1, 2006, p.1115.

COSSON, I. C. O.; OLIVEIRA ,F. N.; ADAN, L. F. Avaliação do conhecimento de medidas preventivas do pé diabético em pacientes de Rio Branco, Acre. Arquivos Brasileiros de Endocrinologia e Metabologia, v. 49, n. 4, 2005, p. 548-556. 